Pacific Journal of Mathematic 


\section{ON THE CLOSED IMAGES OF PARACOMPLEXES}

\section{TAKEMI MizoKaMI}

\section{Let $X$ be an image of a paracomplex by a closed mapping.} Then the following are proved: (1) $X$ is an $M_{1}$-space, and this solves positively the problem proposed by J. Nagata, (2) $\operatorname{dim} X=$ Ind $X$, and (3) $\operatorname{dim} X \leqq n$ if and only if there exists a $\sigma$-closure-preserving base $\mathscr{W}$ for $X$ such that $\operatorname{dim} B(W) \leqq n-1$ for every $W \in \mathscr{W}$.

1. Introduction. J. Nagata in [5] proved that every paracomplex is an $M_{1}$-space in the sense of J. G. Ceder [2], and proposed the problem whether every closed image of a paracomplex is an $M_{1}$-space or not [5, Problem 1]. As noted there, the closed image of a paracomplex is at least stratifiable in the sense of Borges [1]. It is well known that every closed image of a metric space is an $M_{1}$-space [6]. Since a metric space is a 0 -paracomplex, it seems to be natural to propose the problem.

The author proves in this paper that every closed image of a paracomplex is an $M_{1}$-space, and therefore the above problem is solved positively. The point of the proof is to use the notion of uniformly approaching anti-covers, which is first introduced by K. Nagami [4]. The importance of this notion consists in the fact that this property is hereditary with respect to closed mappings. Moreover the author gives two results on dimension theory, one of which is that the coincidence theorem for dim and Ind holds for a closed image of a paracomplex, and the other is that if $X$ is a closed image of a paracomplex, then $\operatorname{dim} X \leqq n$ if and only if there exists a $\sigma$-closure-preserving base $\mathscr{W}$ for $X$ such that $\operatorname{dim} B(W) \leqq n-1$ for every $W \in \mathscr{W}^{-}$, though it is proved by J. Nagata there for a paracomplex itself.

All spaces are assumed to be Hausdorff. All mappings are assumed to be continuous, and onto unless the contrary is stated explicitly. A closed image of a space means the image by a closed mapping. $N$ always denotes the positive integers.

2. Definitions and lemmas. Let $H$ be a closed set of a space $X$ and $f$ a mapping of $H$ into a space $Y$. The quotient space $Z=$ $X \cup_{f} Y$ obtained from the topological disjoint sum $X \vee Y$ by identifying each $x \in H$ with $f(x)$ is called the adjunction space of $X$ and $Y$. If $p: X \vee Y \rightarrow Z$ is the quotient mapping, then we set $p_{X}=$ $p \mid X$ and $p_{Y}=p \mid Y$, which are used in the sequel without the explanation. 
Definition 1 (D. M. Hyman). A 0-paracomplex is a metric space. Assume that $n$-paracomplexes have been defined for $n \in N$. Then the space $Z=X \mathrm{U}_{f} Y$, where $X$ is a metric space and $Y$ is an $n$-paracomplex, is called an $n+1$-paracomplex.

Definition 2([5, Definition 1]). Let $X=\bigcup_{i=1}^{\infty} X_{i}$, where $\left\{X_{i}\right.$ : $i \in N\}$ is a closed cover of a space $X$ such that $X_{i} \subset X_{i+1}$ for each $i \in N$ and each $X_{i}$ is an $n_{i}$-paracomplex for some $n_{i}$ with $0 \leqq n_{i}<\infty$. If $X$ is dominated by $\left\{X_{i}\right\}$ (namely $F \subset X$ is closed in $X$ if and only if $F \cap X_{i}$ is closed in $X_{i}$ for every $i \in N$ ), then $X$ is called a paracomplex.

Definition 3 ([4, Definitions 1.1 and 4.4]). Let $X$ be a space and $F$ a closed set of $X$. An open cover of $X-F$ is said to be an anti-cover of $F$ in $X$. An anti-cover $\mathscr{C}$ is said to be approaching to $F$ in $X$ if for each neighborhood $U$ of $F$ in $X, \overline{S(X-U, \mathscr{U})} \cap$ $F=\varnothing$. An anti-cover $\mathscr{C}$ is said to be uniformly approaching to $F$ in $X$ if for every open set $U$ of $X, \overline{S(X-U, \mathscr{U})} \cap F \cap U=\varnothing$.

The following are known and used later.

Fact 1([4, Remark 4.5(2)]). Every closed set of a metric space has a uniformly approaching anti-cover.

Fact 2 ([4, Theorem 1.3]). Let $X$ be a hereditarily paracompact space and $F$ a closed set of $X$. If $F$ has an approaching anti-cover, then $F$ has a closure-preserving open neighborhood base.

Lemma 1. Let $f: X \rightarrow Y$ be a closed mapping. Let $\mathscr{Q}=\left\{U_{\alpha}\right.$ : $\alpha \in A\}$ be a locally finite open collection of $X$. Let $\Delta$ be the totality of subsets $\delta$ of $A$ and set $V_{o}=\bigcup\left\{U_{\alpha}: \alpha \in \delta\right\}, \delta \in \Delta$. Then $\left\{f\left(X-V_{\delta}\right)\right.$ : $\delta \in \Delta\}$ is closure-preserving in $Y$.

Proof. Let $\Delta_{0}$ be an arbitrary subset of $\Delta$ and suppose $x \notin$ $f\left(X-V_{\delta}\right)$ for every $\delta \in \Delta_{0}$. This implies $f^{-1}(x) \subset V_{\delta}$ for every $\delta \in \Delta_{0}$. For each $y \in f^{-1}(x)$, take an open neighborhood $N(y)$ of $y$ such that $N(y) \subset V_{\delta}$ for every $\delta \in \Delta_{0}$. Set

$$
N=\bigcup\left\{N(y): y \in f^{-1}(x)\right\}, N^{*}=Y-f(X-N) .
$$

Then $N^{*}$ is an open neighborhood of $x$ such that $N^{*} \cap f\left(X-V_{o}\right)=\phi$ for every $\delta \in \Delta_{0}$. This implies $x \notin \overline{\mathrm{U}\left\{f\left(X-V_{\hat{o}}\right): \delta \in \Delta_{0}\right\}}$.

Lemma 2. Let $f: X \rightarrow Y$ be a closed mapping of a hereditarily 
paracompact space $X$ onto $Y$, and $F$ a closed set of $Y$. If $f^{-1}(F)$ has a uniformly approaching anti-cover in $X$, then so does $F$ in $Y$.

Proof. Let $\mathscr{C}=\left\{U_{\alpha}: \alpha \in A\right\}$ be a uniformly approaching anticover of $F$ in $X$ and $\Delta$ the totality of subsets $\delta$ of $A$. Set $V_{\delta}=$ $\bigcup\left\{U_{\alpha}: \alpha \in \delta\right\}$ for each $\delta \in \Delta$. Since $X$ is hereditarily paracompact, we can assume that $\mathscr{C}$ is locally finite in $X-f^{-1}(F)$. For each point $y$ of $Y-F$, define the following:

$$
\begin{gathered}
W(y)=Y-\left[( \mathbf { U } \{ f ( \overline { V } _ { \tilde { \delta } } ) : y \notin f ( \overline { V } _ { \delta } ) \} ) \cup \left(\bigcup \left\{f\left(X-V_{\delta}\right):\right.\right.\right. \\
\left.\left.\left.y \notin f\left(X-V_{\delta}\right)\right\}\right)\right] .
\end{gathered}
$$

Observe $W(y) \cap F=\varnothing$ for every $y \in Y-F$. Since $\left\{f\left(\bar{V}_{\delta}\right): \delta \in \Delta\right\}$ and $\left\{f\left(X-V_{\tilde{o}}\right): \delta \in \Delta\right\}$ are closure-preserving in $Y-F$ by Lemma 1, $W(y)$ is an open neighborhood of $y$ in $Y$. Therefore $\mathscr{W}=\{W(y)$ : $y \in Y-F\}$ is an anti-cover of $F$ in $Y$. We shall show that $\mathscr{W}$ is uniformly approaching to $F$ in $Y$. Let $W$ be an open set of $Y$. Since $\mathscr{C}$ is uniformly approaching, there exist $\delta_{1}, \delta_{2} \in \Delta$ such that

$$
N_{i}=\left(f^{-1}(W) \cap f^{-1}(F)\right) \cup V_{\delta_{i}}, \quad i=1,2,
$$

are open sets of $X$ such that

$$
N_{1} \subset f^{-1}(W), \quad N_{2} \cap S\left(X-f^{-1}\left(N_{1}^{*}\right), \mathscr{C}\right)=\dot{\phi},
$$

where $N_{1}^{*}=Y-f\left(X-N_{1}\right)$. It follows from the definition of $W(y)$ that if $y \in Y-\left(f\left(X-V_{\delta_{1}}\right) \cup F\right)$, then $W(y) \subset W$. Therefore if $W(y) \cap(Y-W) \neq \varnothing$ for $y \in Y-F$, then $y \in f\left(X-V_{\delta_{1}}\right)$. This implies $y \notin f\left(\bar{V}_{\bar{o}_{2}}\right)$. Thus we have $W(y) \cap f\left(\bar{V}_{\bar{o}_{2}}\right)=\varnothing$. If we set ${N_{2}^{*}}^{*}=Y-$ $f\left(X-N_{2}\right)$, then $N_{2}^{*}$ is an open neighborhood of $F \cap W$ in $Y$ such that $N_{2}^{*} \cap W(y)=\varnothing$. Hence we have $S(Y-W, \mathscr{W}) \cap N_{2}^{*}=\varnothing$. This completes the proof.

Definition 4. A space $X$ is said to have the property $(P)$ if for every closed set $F$ of $X$ and for every open set $V$ of $F$, there corresponds an open set $V^{\prime}$ of $X$ such that $V^{\prime} \cap F=V$ and $V^{\prime} \cap$ $W^{\prime}=\varnothing$ if $V, W$ are disjoint open sets of $F$. Such an open extension $V^{\prime}$ of $V$ is said to be a special extension of $V$ to $X$.

Lemma 3. Every n-paracomplex has the property $(P)$.

Proof. First we consider the case of $n=0$. Let $F$ be a closed set of a metric space $(X, d)$ and $V$ an open set of $F$. For every point $x \in V$, choose a positive number $\varepsilon=\varepsilon(x)$ such that $S_{\varepsilon}(x) \cap F \subset V$, where $S_{\varepsilon}(x)$ denotes the $\varepsilon$-neighborhood of $x$ with respect to $d$. Set 


$$
V^{\prime}=\bigcup\left\{S_{\varepsilon / 2}(x): x \in V\right\} .
$$

Then $V^{\prime}$ is easily seen to be a special extension of $V$ to $X$. We consider the general case. Assume that every $n$-paracomplex has the property $(P)$. Let $Z=X \bigcup_{f} Y$ be an $n+1$-paracomplex stated in Definition 1. Let $F$ be a closed set of $Z$ and $V$ an open set of $F$. Since $Y$ has $(P)$ by the induction assumption, $p_{Y}^{-1}(V)$ has a special extension $\widetilde{V}$ to $Y$. Let $d$ be a compatible metric on $X$. Choose for each point $x$ of $p_{X}^{-1}(V \cup p(\tilde{V}))$ a positive number $\varepsilon=\varepsilon(x)$ such that

$$
S_{\varepsilon}(x) \cap\left(H \cup p_{\bar{X}}^{-1}\left(F^{\prime}\right)\right) \subset p_{\bar{X}}^{-1}(V \cup p(\tilde{V})),
$$

where $S_{\varepsilon}(x)$ denotes the $\varepsilon$-neighborhood of $x$ with respect to $d$. Set

$$
V^{\prime}=p(\tilde{V}) \cup p\left(\bigcup\left\{S_{\varepsilon / 2}(x): x \in p_{X}^{-1}(V \cup p(\tilde{V}))\right\}\right) \text {. }
$$

Then by a routine check $V^{\prime}$ is shown to be a special extension of $V$ to $Z$.

LEMMA 4. Every closed image of a space with the property $(P)$ also has $(P)$.

Proof. Let $f: X \rightarrow Y$ be a closed mapping of a space with $(P)$ onto $Y$. Let $F$ be a closed set of $Y$ and $V$ an open set of $F$. There exists a special extension $f^{-1}(V)^{\prime}$ of $f^{-1}(V)$ to $X$. Set $V^{\prime}=$ $Y-f\left(X-f^{-1}(V)^{\prime}\right)$. Then $V^{\prime}$ is an open set of $Y$ such that $V^{\prime} \cap$ $F=V$. It is obvious that if $V, W$ are disjoint open sets of $F$, then $V^{\prime} \cap W^{\prime}=\varnothing$. Hence $Y$ has the property $(P)$.

Definition 5. A space $X$ is said to be cp-expandable if the following condition $(C P)$ is satisfied:

$(C P)$ Let $F$ be an arbitrary closed set of $X$ and $\mathscr{U}=\left\{U_{\alpha}: \alpha \in\right.$ $A$ ) a closure-preserving open collection of $F$. Then for each $\alpha \in A$ there exists an open collection $\left\{U_{\alpha \beta}: \beta \in B_{\alpha}\right\}$ of $X$ with $U_{\alpha \beta} \cap F=U_{\alpha}$ for every $\beta \in B_{a}$, which satisfies the following (1) and (2):

(1) $\mathscr{\mathscr { V }}=\left\{U_{\alpha \beta}: \beta \in B_{\alpha}, \alpha \in A\right\}$ is closure-preserving in $X$.

(2) If $U_{\alpha}=W \cap F$ for an open set $W$ of $X$ and for $\alpha \in A$, then $U_{\alpha \beta} \subset W$ for some $\beta \in B_{\alpha}$.

In the next two lemmas, we shall show that every closed image of an $n$-paracomplex is $c p$-expandable, and this fact plays an important role when Theorem 1 is proved.

LeMma 5. Every closed image $T$ of an n-paracomplex has a collection $\left\{T_{i}: i=1, \cdots, k\right\}$ of $T$ satisfying the following:

(1) $T=\bigcup_{i=1}^{k} T_{i}, T_{i} \cap T_{j}=\varnothing$ for each pair $i, j$ with $i \neq j$ and 
each $T_{i}^{\prime}=T_{1} \cup \cdots \cup T_{i}$ is closed in $T$.

(2) If $F$ is a closed set of $T$, then $F$ and each $F \cup T_{i}^{\prime}, i=1, \cdots$, $k-1$, have uniformly approaching anti-covers in $F \cup T_{1}$ and $F \cup T_{i+1}$, respectively.

Proof. Part 1. We shall show that every $n$-paracomplex $Z$ has the collection $\left\{Z_{i}: i=1, \cdots, k\right\}$ of subsets of $Z$ satisfying (1) and (2) in Lemma 5. A 0 -paracomplex $Z$ has obviously a collection $\left\{Z_{1}\right\}=\{Z\}$ satisfying (1) and (2) by virtue of Fact 1 . Assume that every $n$-paracomplex has such a collection. Suppose that we are given $Z=X \mathbf{U}_{f} Y$, where $Y$ is an $n$-paracomplex and $f$ is a mapping of a closed set $H$ of a metric space $X$ into $Y$. By the induction assumption $Y$ has a collection $\left\{Y_{1}, \cdots, Y_{k}\right\}$ satisfying (1) and (2). Set

$$
Z_{1}=p\left(Y_{1}\right), \cdots, Z_{k}=p\left(Y_{k}\right), Z_{k+1}=Z-p(Y) .
$$

Then $\left\{Z_{1}, \cdots, Z_{k+1}\right\}$ obviously satisfies (1) in Lemma 5 . Let $F$ be a closed set of $Z$. By Fact $1, H \cup p_{X}^{-1}(F)$ has a uniformly approaching anti-cover $\mathscr{V}$ in $X$. Let $\mathscr{C}_{0}, \mathscr{C}_{i}, i=1, \cdots, k-1$, be uniformly approaching anti-covers of $p_{Y}^{-1}(F), p_{Y}^{-1}(F) \cup\left(Y_{1} \cup \cdots \cup Y_{i}\right)$ in $p_{Y}^{-1}(F) \cup$ $Y_{1}, p_{Y}^{-1}(F) \cup\left(Y_{1} \cup \cdots \cup Y_{i+1}\right)$, respectively. Set

$$
\mathscr{W}_{0}=p\left(\mathscr{U}_{0}\right), \quad \mathscr{W}_{1}=p\left(\mathscr{U}_{1}\right), \cdots, \quad \mathscr{W}_{k-1}=p\left(\mathscr{U}_{k-1}\right) .
$$

Then obviously $\mathscr{Y}_{0}, \mathscr{W}_{i}, i=1, \cdots, i-1$, are uniformly approaching anti-covers of $F, F \cup\left(Z_{1} \cup \cdots \cup Z_{i}\right)$ in $F \cup Z_{1}, F \cup\left(Z_{1} \cup \cdots \cup Z_{i+1}\right)$, respectively. It is obvious that $\mathscr{Y}_{k}=p(\mathscr{Y})$ is a uniformly approaching anti-cover of $F \cup\left(Z_{1} \cup \cdots \cup Z_{k}\right)=F \cup p(Y)$ in $Z$.

Part 2. Let $g: Z \rightarrow T$ be a closed mapping of an $n$-paracomplex $Z$ onto $T$. By Part 1 , there exists a collection $\left\{Z_{1}, \cdots, Z_{t}\right\}$ of subsets of $Z$ satisfying (1) and (2). Set

$$
\begin{gathered}
T_{1}=g\left(Z_{1}\right), T_{i}=g\left(Z_{i}\right)-\left(g\left(Z_{1}\right) \cup \cdots \cup g\left(Z_{i-1}\right)\right), \\
i=2, \cdots, t .
\end{gathered}
$$

Then obviously $\left\{T_{i}: i=1, \cdots, t\right\}$ satisfies (1). Let $F$ be a closed set of $T$. Set

$$
g_{i}=g \mid P_{\imath}, P_{\imath}=\left(Z_{1} \cup \cdots \cup Z_{i}\right) \cup g^{-1}(F), i=1, \cdots, t .
$$

Then $g_{i}: P_{i} \rightarrow F \cup\left(T_{1} \cup \cdots \cup T_{i}\right)$ is a closed mapping. Since $g_{1}^{-1}(F)$ has a uniformly approaching anti-cover in $P_{1}$, by Lemma 2 so does $F$ in $F \cup T_{1}$. Observe that $g_{i+1}^{-1}\left(F \cup\left(T_{1} \cup \cdots \cup T_{i}\right)\right)$ is a closed set of $P_{\imath+1}$ containing $Z_{1} \cup \cdots \cup Z_{i}$, and therefore by (2) for $\left\{Z_{1}, \cdots, Z_{t}\right\}$ it has a uniformly approaching anti-cover in $P_{i+1}$. Consequently by Lemma $2 F \cup\left(T_{1} \cup \cdots \cup T_{i}\right), i=1, \cdots t-1$, has a uniformly approaching anti-cover in $F \cup\left(T_{1} \cup \cdots \cup T_{i+1}\right)$. This completes the proof. 
Lemma 6. Every closed image of an n-paracomplex is cp-expandable.

Proof. Let $f: Z \rightarrow T$ be a closed mapping of an $n$-paracomplex $Z$ onto $T$. Then by Lemma 5 there exists a collection $\left\{T_{1}, \cdots, T_{k}\right\}$ satisfying (1) and (2) in Lemma 5 . Let $F$ be a closed set of $T$ and $\mathscr{C}=\left\{U_{\alpha}: \alpha \in A\right\}$ a closure-preserving open collection of $F$. By (2) $F, F \cup\left(T_{1} \cup \cdots \cup T_{i}\right), i=1, \cdots, k-1$, have uniformly approaching anti-covers $\mathscr{C}_{0}, \mathscr{C}_{i}$ in $F \cup T_{1}, F \cup\left(T_{1} \cup \cdots \cup T_{i+1}\right)$, respectively. Since $T$ is hereditarily paracompact, we can assume that $\mathscr{C}_{0}=$ $\left\{U_{0 \alpha}: \alpha \in A_{0}\right\}, \mathscr{U}_{i}=\left\{U_{i \alpha}: \alpha \in A_{i}\right\}, i=1, \cdots, k-1$, are locally finite in $T_{1}-F, T_{i+1}-F$, respectively. For each $\alpha \in A_{0}$, let $\Delta(\alpha)$ be the totality of subsets $\delta_{0}$ of $A_{0}$ such that

$$
U_{\alpha}\left(\delta_{0}\right)=U_{\alpha} \cup\left(\bigcup\left\{U_{0 \alpha}: \alpha \in \delta_{0}\right\}\right)
$$

is an open set of $F \cup T_{1}$ such that $U_{\alpha}\left(\delta_{0}\right) \subset U_{\alpha}^{\prime}$, where $U_{\alpha}^{\prime}$ is a special extension of $U_{\alpha}$ to $T$, the existence of which is assured by Lemmas 3 and 4 . For each $\delta_{0} \in \Delta(\alpha)$, let $\Delta\left(\alpha, \delta_{0}\right)$ be the tatality of subsets $\delta_{1}$ of $A_{1}$ such that

$$
U_{\alpha}\left(\delta_{0}, \delta_{1}\right)=U_{\alpha}\left(\delta_{0}\right) \cup\left(\cup\left\{U_{1 \alpha}: \alpha \in \delta_{1}\right\}\right)
$$

is an open set of $F \cup\left(T_{1} \cup T_{2}\right)$ such that

$$
U_{\alpha}\left(\delta_{0}, \delta_{1}\right) \subset U_{\alpha}^{\prime} \cap U_{\alpha}\left(\delta_{0}\right)^{\prime},
$$

where $U_{\alpha}\left(\delta_{0}\right)^{\prime}$ is a special extension of $U_{\alpha}\left(\delta_{0}\right)$ to $T$. Repeating this process, we reach an open set of $T$

$$
U_{\alpha}\left(\delta_{0}, \cdots, \delta_{k-1}\right)=U_{\alpha}\left(\delta_{0}, \cdots, \delta_{k-2}\right) \cup\left(\bigcup\left\{U_{k-1 \alpha}: \alpha \in \delta_{k-1}\right\}\right)
$$

for $\delta_{k-1} \in \Delta\left(\alpha, \delta_{0}, \cdots, \delta_{k-2}\right)$. For each $\beta=\left(\delta_{0}, \delta_{1}, \cdots, \delta_{k-1}\right)$, we set $U_{\alpha \beta}=U_{\alpha}\left(\delta_{0}, \cdots, \delta_{k-1}\right)$ and consider the collection

$$
\begin{aligned}
\mathscr{V}= & \left\{U_{\alpha \beta}: \beta=\left(\delta_{0}, \cdots, \delta_{k-1}\right), \delta_{0} \in \Delta(\alpha),\right. \\
& \left.\delta_{1} \in \Delta\left(\alpha, \delta_{0}\right), \cdots, \delta_{k-1} \in \Delta\left(\alpha_{0}, \cdots, \delta_{k-2}\right), \alpha \in A\right\} .
\end{aligned}
$$

Then obviously $U_{\alpha \beta} \cap F=U_{\alpha}$ for each $\alpha, \beta$. To show that $\mathscr{y}$ is closure-preserving in $T$, let $M$ be an arbitrary subset of $\{(\alpha, \beta): \beta=$ $\left.\left(\delta_{0}, \cdots, \delta_{k-1}\right), \quad \delta_{0} \in \Delta(\alpha), \cdots, \delta_{k-1} \in \Delta\left(\alpha, \delta_{0}, \cdots, \delta_{k-2}\right), \quad \alpha \in A\right\}$. Suppose $x \notin \bar{U}_{\alpha \beta}$ for every $(\alpha, \beta) \in M$. If $x \in F$, then there exists an open neighborhood $N$ of $x$ in $F$ such that $N \cap U_{\alpha}=\varnothing$ for every $\alpha \in A$ with $(\alpha, \beta) \in M$. Let $N^{\prime}$ be a special extension of $N$ to $T$. Then $U_{\alpha \beta} \cap N^{\prime}=\varnothing$ for every $(\alpha, \beta) \in M$ by the property of special extensions. If $x \notin F$, then $x \in T_{i}-F$ for some $i$. Since $\mathscr{C}_{i-1}$ is locally finite at $x$, there exists an open neighborhood $N$ of $x$ in $F \cup$ 
$\left(T_{1} \cup \cdots \cup T_{i}\right)$ such that $N \cap U_{\alpha}\left(\delta_{0}, \cdots, \delta_{i-1}\right)=\varnothing$ for every $(\alpha, \beta) \in M$, $\beta=\left(\delta_{0}, \cdots, \delta_{k-1}\right)$. Let $N^{\prime}$ be a special extension of $N$ to $T$, if $i \neq k$. If $i=k$, let $N^{\prime}=N$. Since $U_{\alpha \beta} \subset U_{\alpha}\left(\delta_{0}, \cdots, \delta_{i-1}\right)^{\prime}$ and $U_{\alpha}\left(\delta_{0}, \cdots\right.$, $\left.\delta_{i-1}\right)^{\prime} \cap N^{\prime}=\varnothing$ for every $(\alpha, \beta) \in M, \beta=\left(\delta_{0}, \cdots, \delta_{i-1}\right), N^{\prime} \cap U_{\alpha \beta}=\varnothing$ for every $(\alpha, \beta) \in M$. Therefore in either case $p \notin \overline{\mathrm{U}\left\{U_{\alpha \beta}:(\alpha, \beta) \in M\right\}}$, proving that $\mathscr{V}$ is closure-preserving in $T$. Let $U$ be an open set of $T$ such that $U \cap F=U_{\alpha}$ for some $\alpha$. Then since $\mathscr{W}_{1}$ is uniformly approacing in $F \cup T_{1}$, there exists a $\delta_{0} \in \Delta(\alpha)$ such that $U_{\alpha}\left(\delta_{0}\right) \subset U$. Similarly we can choose $\delta_{1} \in \Delta\left(\alpha, \delta_{0}\right)$ such that $U_{\alpha}\left(\delta_{0}, \delta_{1}\right) \subset U$. Repeating this process, we get $\beta=\left(\delta_{0}, \cdots, \delta_{k-1}\right)$ such that $U_{\alpha \beta} \subset U$. This completes the proof.

As stated in the introduction, every closed image of an $n$-paracomplex is at least stratifiable, and consequently it is a $\sigma$-space, that is, it has a $\sigma$-discrete closed network. In the next proof, a collection $\mathscr{F}$ of subsets of a space $T$ is said to be local network of a point $x \in T$ if $x \in U$ with $U$ open in $T$ implies $x \in F \subset U$ for some $F \in \mathscr{F}$.

LeMma 7. Every closed image of an n-paracomplex is an $M_{1^{-}}$ space.

Proof. Since every closed image of a 0-paracomplex is a Lašnev space, it is an $M_{1}$-space, [6]. Assume that every closed image of an $n$-paracomplex is an $M_{1}$-space. Let $g: Z=X \bigcup_{f} Y \rightarrow T$ be a closed mapping of an $n+1$-paracomplex $Z$ onto $T$, where $X$ is a metric space, $Y$ is an $n$-paracomplex and $f$ is a mapping of a closed set $H$ into $Y$. By the induction assumption, $g(p(Y))$ is an $M_{1}$-space. Therefore $g(p(Y))$ has a $\sigma$-closure-preserving base $\mathscr{C}=\left\{U_{\alpha}: \alpha \in A\right\}$. By Lemma $6 T$ is $c p$-expandable and $g(p(Y))$ is closed in $T$. We apply the $c p$-expandability of $T$ to $\mathscr{C}$ to get a $\sigma$-closure-preserving open collection $\mathscr{C}_{0}=\left\{U_{\alpha \beta}: \beta \in B_{\alpha}, \alpha \in A\right\}$ of $T$ such that

$$
U_{\alpha \beta} \cap g(p(Y))=U_{\alpha} \text { for } \beta \in B_{\alpha}, \alpha \in A,
$$

and such that if $U \cap g(p(Y))=U_{\alpha}$ for an $\alpha \in A$ and for an open set $U$ of $T$, then $U_{\alpha \beta} \subset U$ for some $\beta \in B_{\alpha}$. Let $F$ be an arbitrary closed set of $T$ such that $F \cap g(p(Y))=\varnothing$. Then it is obvious that $g^{-1}(F)$ has a uniformly approaching anti-cover in $Z$. Thus by Lemma $2, F$ has a (uniformly) approaching anti-cover in $T$. Therefore by Fact $2 F$ has a closure-preserving open neighborhood base $\mathscr{C}(F)$ in $T$. Let $\mathscr{F}=\bigcup_{i=1}^{\infty} \mathscr{F}_{i}$, with each $\mathscr{F}_{i}$ discrete in $T$, be a closed collection of $T$, which is a local network of each point of $T-g(p(Y))$ in $T$. By the paracompactness of $T$, there exists for 
each $i \in N$ a discrete open collection $\left\{H(F): F \in \mathscr{F}_{i}\right\}$ such that $F \subset$ $H(F)$ and $H(F) \cap g(p(Y))=\varnothing$ for every $F \in \mathscr{F}_{i}$. Set

$$
\begin{aligned}
& \mathscr{U}^{\prime}(F)=\{U \in \mathscr{U}(F): U \subset H(F)\}, \\
& \mathscr{U}_{1}=\bigcup\left\{\mathscr{U}^{\prime}(F): F \in \mathscr{F}\right\}, \\
& \mathscr{W}=\mathscr{U}_{0} \cup \mathscr{U}_{1} .
\end{aligned}
$$

Since $\bigcup\left\{\mathscr{C}^{\prime}(F): F \in \mathscr{F}_{i}\right\}$ is closure-preserving in $T$, $\mathscr{W}^{-}$is a $\sigma$-closurepreserving open collection. It is obvious that $\mathscr{W}$ is a base for $T$. Hence $T$ is an $M_{1}$-space.

\section{Theorems.}

THEOREM 1. Every closed image of a paracomplex is an $M_{1}$ space.

Proof. Let $f: X \rightarrow Y$ be a closed mapping of a paracomplex $X$ onto $Y$. Let $\left\{X_{i}: i \in N\right\}$ be a closed cover of $X$ stated in Definition 2. Since $X_{1}$ is an $n_{1}$-paracomplex, by Lemma 7 there exists a $\sigma$-closure-preserving base $\mathscr{U}_{\mathscr{C}}=\bigcup_{m=1}^{\infty} \mathscr{U}_{m}$ for $f\left(X_{1}\right)$, where each $\mathscr{U}_{m}=$ $\left\{U(\alpha): \alpha \in A_{m}\right\}$ is a closurepreserving open collection of $f\left(X_{1}\right)$. Observe that $f\left(X_{2}\right)$ is $c p$-expandable by Lemma 6 and that $f\left(X_{1}\right)$ is closed in $f\left(X_{2}\right)$. Therefore for each $\alpha_{1} \in A_{m}$, there exists an open collection $\left\{U\left(\alpha_{1}, \alpha_{2}\right): \alpha_{2} \in A_{m}\left(\alpha_{1}\right)\right\}$ of $f\left(X_{2}\right)$ such that

$$
U\left(\alpha_{1}, \alpha_{2}\right) \cap f\left(X_{1}\right)=U\left(\alpha_{1}\right), U\left(\alpha_{1}, \alpha_{2}\right) \subset U\left(\alpha_{1}\right)^{\prime},
$$

where $U\left(\alpha_{1}\right)^{\prime}$ is a special extension of $U\left(\alpha_{1}\right)$ to $f\left(X_{2}\right)$ and such that and

(1) $\left\{U\left(\alpha_{1}, \alpha_{2}\right): \alpha_{2} \in A_{m}\left(\alpha_{1}\right), \alpha_{1} \in A_{m}\right\}$ is closure-preserving in $f\left(X_{2}\right)$,

(2) if $V$ is an open set of $f\left(X_{2}\right)$ with $V \cap f\left(X_{1}\right)=U\left(\alpha_{1}\right)$ for an $\alpha_{1} \in A_{m}$, then $U\left(\alpha_{1}, \alpha_{2}\right) \subset V$ for some $\alpha_{2} \in A_{m}\left(\alpha_{1}\right)$.

Again by the $c p$-expandability of $f\left(X_{3}\right)$, we define the following collection: For each $\left(\alpha_{1}, \alpha_{2}\right)$ with $\alpha_{2} \in A_{m}\left(\alpha_{1}\right), \alpha_{1} \in A_{m}$, there corresponds an open collection $\left\{U\left(\alpha_{1}, \alpha_{2}, \alpha_{3}\right): \alpha_{3} \in A_{m}\left(\alpha_{1}, \alpha_{2}\right)\right\}$ of $f\left(X_{3}\right)$ such that

$$
\begin{aligned}
& U\left(\alpha_{1}, \alpha_{2}, \alpha_{3}\right) \cap f\left(X_{2}\right)=U\left(\alpha_{1}, \alpha_{2}\right), \\
& U\left(\alpha_{1}, \alpha_{2}, \alpha_{3}\right) \subset U\left(\alpha_{1}, \alpha_{2}\right)^{\prime},
\end{aligned}
$$

where $U\left(\alpha_{1}, \alpha_{2}\right)^{\prime}$ is a special extension of $U\left(\alpha_{1}, \alpha_{2}\right)$ to $f\left(X_{3}\right)$, and such that

$$
\text { the collection }\left\{U\left(\alpha_{1}, \alpha_{2}, \alpha_{3}\right): \alpha_{3} \in A_{m}\left(\alpha_{1}, \alpha_{2}\right)\right. \text {, }
$$

$\left.\alpha_{2} \in A_{m}\left(\alpha_{1}\right), \alpha_{1} \in A_{m}\right\}$ is closure-preserving in $f\left(X_{3}\right)$, and 
$(2)^{\prime}$ if $V$ is an open set of $f\left(X_{3}\right)$ with $V \cap f\left(X_{2}\right)=U\left(\alpha_{1}, \alpha_{2}\right)$ for an $\alpha_{2} \in A_{m}\left(\alpha_{1}\right), \alpha_{1} \in A_{m}$, then $U\left(\alpha_{1}, \alpha_{2}, \alpha_{3}\right) \subset V$ for some $\alpha_{3} \in A_{m}\left(\alpha_{1}, \alpha_{2}\right)$. Repeating this process, for each $i \in N$ we get a closure-preserving open collection $\left\{U\left(\alpha_{1}, \cdots, \alpha_{\imath}\right): \alpha_{i} \in A_{m}\left(\alpha_{1}, \cdots, \alpha_{i-1}\right), \cdots, \alpha_{1} \in A_{m}\right\}$, which satisfies the conditions corresponding to (1), (1)' and (2), (2)'. For each sequence $\mu=\left(\alpha_{1}, \alpha_{2}, \cdots\right)$ with $\alpha_{1} \in A_{m}, \alpha_{2} \in A_{m}\left(\alpha_{1}\right), \cdots$, we set

$$
U_{\mu}=\bigcup_{i=1}^{\infty} U\left(\alpha_{1}, \cdots, \alpha_{i}\right) \text {. }
$$

Set

$$
\begin{aligned}
& \mathscr{V}(m)=\left\{U_{\mu}: \mu=\left(\alpha_{1}, \alpha_{2}, \cdots\right), \alpha_{1} \in A_{m}, \alpha_{2} \in A_{m}\left(\alpha_{1}\right), \cdots\right\}, \\
& \mathscr{V}_{1}=\mathbf{U}\{\mathscr{V}(m): m \in N\} .
\end{aligned}
$$

First, it is shown that each $\mathscr{\mathscr { C }}(m)$ is closure-preserving open collection of $Y$. Indeed, take a $U_{\mu} \in \mathscr{W}^{-}(m), \mu=\left(\alpha_{1}, \alpha_{2}, \cdots\right)$. Then $U_{\mu}$ is open in $Y$ because

$$
U_{\mu} \cap f\left(X_{i}\right)=U\left(\alpha_{1}, \cdots, \alpha_{\imath}\right), i \in N
$$

is open in $f\left(X_{i}\right)$ and $\left\{f\left(X_{\imath}\right): i \in N\right\}$ dominates $Y$. Let $M$ be an arbitrary subset of $\left\{\mu=\left(\alpha_{1}, \alpha_{2}, \cdots\right): \quad \alpha_{1} \in A_{m}, \quad \alpha_{2} \in A_{m}\left(\alpha_{1}\right), \quad \alpha_{3} \in\right.$ $\left.A_{m}\left(\alpha_{1}, \alpha_{2}\right), \cdots\right\}$ and suppose $x \notin \bar{U}_{\mu}$ for every $\mu \in M$. There exists an $i \in N$ such that $x \in f\left(X_{i}\right)$. Since $\left\{U\left(\alpha_{1}, \cdots, \alpha_{i}\right): \mu=\left(\alpha_{1}, \alpha, \cdots\right) \in\right.$ $M\}$ is closure-preserving in $f\left(X_{i}\right)$ by (1), (1)', .., there exists an open neighborhood $N$ of $x$ in $f\left(X_{i}\right)$ such that

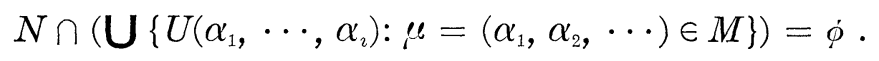

Let $N_{i+1}$ be a special extension of $N$ to $f\left(X_{i+1}\right)$. For each $j \geqq 2$, let $N_{i+j}$ be a special extension of $N_{i+j-1}$ to $f\left(X_{i+j}\right)$. Set $E(N)=$ $\bigcup_{j=1}^{\infty} N_{\imath+j}$. Then $E(N)$ is an open neighborhood of $x$ in $Y$ such that

$$
E(N) \cap\left(\bigcup\left\{U_{\mu}: \mu \in M\right\}\right)=\dot{\phi} .
$$

Therefore $\left.x \notin \overline{\mathrm{U}\left\{\bar{U}_{\ell}: \mu \in M\right.}\right\}$, which proves that $\mathscr{W}^{-}(m)$ is closurepreserving in $Y$. Secondly, we show that $\mathscr{W}_{1}$ is a local base of each point $x$ of $f\left(X_{1}\right)$ in $Y$. To see this, let $x \in V$ for an open set $V$ of $Y$ and a point $x$ of $f\left(X_{1}\right)$. Since $\mathscr{U}$ is a base for $f\left(X_{1}\right)$, there exists an $\alpha_{1} \in A_{m}, m \in N$, such that $x \in U\left(\alpha_{1}\right) \subset V$. By (2) and (2)', there exist $\alpha_{2} \in A_{m}\left(\alpha_{1}\right)$ and $\alpha_{3} \in A_{m}\left(\alpha_{1}, \alpha_{2}\right)$ such that $x \in U\left(\alpha_{1}, \alpha_{2}, \alpha_{3}\right) \subset$ $V$. Inductively we can get an $\alpha_{i}$ such that $x \in U\left(\alpha_{1}, \cdots, \alpha_{\imath}\right) \subset V$. Therefore $x \in U_{\mu} \subset V$ for $\mu=\left(\alpha_{1}, \alpha_{2}, \cdots\right)$. By the same way as $\mathscr{V}_{1}$, we can construct a $\sigma$-closure-preserving open collection $\mathscr{W}_{2}$ of $Y$ which is a local base of each point of $f\left(X_{i}\right)$ in $Y$. Then $\mathscr{W}=$ $\bigcup_{i=1}^{\infty} \mathscr{V}_{i}^{i}$ is a $\sigma$-closure-preserving base for $Y$, proving that $Y$ is an $M_{1}$-space. 
We consider the dimension for the closed image of a paracomplex. To state it, we prepare some definitions and some lemmas.

Definition 6 ([4, Definition 3.4]). A collection $\mathscr{T}=\left\{\left(H_{\alpha}, K_{\alpha}\right)\right.$ : $\alpha \in A\}$ of disjoint pairs of closed sets of a space $X$ is said to determine Ind $X$ if there exists a pair $\left(H_{\alpha}, K_{\alpha}\right) \in \mathscr{T}$ such that for each closed set $P$ separating $H_{\alpha}$ and $K_{\alpha}$, Ind $P \geqq \operatorname{Ind} X-1$. Let $M$ be subset of $X$. Then $\mathscr{T}$ is said to determine Ind $M$ if $\mathscr{T} \mid M$ determines Ind $M$.

It is proved in [4, Theorem 4.1] that if $X$ is a paracompact $\sigma$-space which has a countable collection of disjoint pairs of closed sets determining Ind of all closed sets of $X$, then $I n d=\operatorname{dim} X$, and it is also proved in [4, Lemma 3.5] that every $L$-space has such a collection. Since every Lašnev space is an $L$-space, [4, Theorem 1.6], every Lašnev space has such a collection. Since every closed image of a paracomplex is a paracompact $\sigma$-space and is the countable union of closed Lašnev spaces, it has a countable collection of disjoint pairs of closed sets determining Ind of all closed sets. Thus we have:

THEOREM 2. If $X$ is a closed image of a paracomplex, then $\operatorname{dim} X=$ Ind $X$.

Let (A) be the statement that $\operatorname{dim} X \leqq n$ if and only if there exists a $\sigma$-closure-preserving base $\mathscr{W}$ for $X$ such that $\operatorname{dim} B(W) \leqq$ $n-1$ for every $W \in \mathscr{W}$, where $B(W)$ denotes the boundary of $W$ in $X$.

As shown by J. Nagata, if $X$ is a paracomplex, (A) hold for $X$. But we shall show in the next theorem that (A) holds for every closed image of a paracomplex. The proof is given by modifying the argument in Theorem 1.

Definition 7. A space $X$ is said to be $n$-dimensionally $c p-e x-$ pandabe if the following condition $(C P)_{\mathfrak{n}}$ is satisfies:

$(C P)_{n}$ Let $F$ be a closed set of $X$ and $\mathscr{C}=\left\{U_{\alpha}: \alpha \in A\right\}$ a closure-preserving open collection of $F$ such that $\operatorname{dim} B_{F}\left(U_{\alpha}\right) \leqq n-1$ for every $\alpha \in A$, where $B_{F}\left(U_{\alpha}\right)$ denotes the boundary of $U_{\alpha}$ in $F$. Then there exists an open collection $\mathscr{V}=\left\{U_{\alpha \beta}: \beta \in B_{\alpha}, \alpha \in A\right\}$ of $X$ with $U_{\alpha \beta} \cap F=U_{\alpha}$ for every $\beta \in B_{\alpha}, \alpha \in A$, satisfying (1) and (2) stated in Definition 5 and the additional condition (3).

(3) $\operatorname{dim} B\left(U_{\alpha \beta}\right) \leqq n-1$ for every $\beta \in B_{\alpha}, \alpha \in A$.

Lemma 8. Every closed image $T$ with $\operatorname{dim} T \leqq n$ of an $m$ - 
paracomplex is n-dimensionally cp-expandable.

Proof. Suppose that we are given a closed mapping $f$ of an $m$-paracomplex $Z$ onto $T$ with $\operatorname{dim} T \leqq n$. In this proof we use the same denotion as in the proof of Lemma 6 . Let $F$ be a closed set of $T$ and $\mathscr{\mathscr { C }}=\left\{U_{\alpha}: \alpha \in A\right\}$ a closure-preserving open collection of $F$ such that $\operatorname{dim} B_{F}\left(U_{\alpha}\right) \leqq n-1$ for every $\alpha \in A$. Since $T$ satisfies $\operatorname{dim} T \leqq n$, in addition to the assumption stated in the proof of Lemma 6 we can assume that

$$
\operatorname{dim} B_{T_{1}-F}\left(U_{0 \alpha}\right) \leqq n-1, \operatorname{dim} B_{T_{i+1}-F}\left(U_{i \alpha^{\prime}}\right) \leqq n-1
$$

for every $\alpha \in A_{0}$ and for every $\alpha^{\prime} \in A_{i}, i=1, \cdots, k-1$. It follows from an easy check that for each $\beta=\left(\delta_{0}, \cdots, \delta_{k-1}\right)$

$$
B\left(U_{\alpha \beta}\right) \subset B_{F}\left(U_{\alpha}\right) \cup\left(\bigcup_{i=0}^{k-1}\left\{\bigcup_{\alpha \in \delta_{i}} B_{T_{i+1}-F}\left(U_{i \alpha}\right)\right\}\right)
$$

Then by the sum theorem we have

$$
\operatorname{dim} B\left(U_{\alpha \beta}\right) \leqq n-1 \text { for every } \beta .
$$

Thus $(C P)_{n}$ is satisfied.

LEMMA 9. If $T$ is a closed image of an m-paracomplex such that $\operatorname{dim} T \leqq n$, then there exists a $\sigma$-closure-preserving base $\mathscr{W}$ for $T$ such that $\operatorname{dim} B(W) \leqq n-1$ for every $W \in \mathscr{W}$.

Proof. Let $g: Z \rightarrow T$ be a closed mapping of an $m$-paracomplex $Z$ onto $T$. Suppose $\operatorname{dim} T \leqq n$. If $Z$ is a 0 -paracomplex, then $T$ is a Lašnev space and therefore by [3, Theorem 2] there exists a $\sigma$ closure-preserving base with the required property. Assume that every closed image of an $m$-paracomplex has such a base. Let $Z=$ $X \cup_{f} Y$, where $Y$ is an $m$-paracomplex and $X$ is a metric space. By the induction assumption $g(P(Y))$ has a $\sigma$-closure-preserving base $\mathscr{Q}=\left\{U_{\alpha}: \alpha \in A\right\}$ such that $\operatorname{dim} B_{g(p(Y))}\left(U_{\alpha}\right) \leqq n-1$ for every $\alpha \in A$. Since by Lemma $8 T$ is $n$-dimensionally $c p$-expandable, there exists an open collection $\mathscr{U}_{0}=\left\{U_{\alpha \beta}: \beta \in B_{\alpha}, \alpha \in A\right\}$ of $T$ satisfying the following:

(1) $\mathscr{U}_{0}$ is $\sigma$-closure-preserving in $T$.

(2) If $V \cap g(p(Y))=U_{\alpha}$ for an $\alpha \in A$, then $U_{\alpha \beta} \subset V$ for some $\beta \in B_{\alpha}$.

(3) $\operatorname{dim} B\left(U_{\alpha \beta}\right) \leqq n-1$ for every $\beta \in B_{\alpha}, \alpha \in A$. Let $\mathscr{F}=\bigcup_{i=1}^{\infty} \mathscr{F}_{i},\left\{H(F): F \in \mathscr{F}_{i}\right\}$ be the same as in the proof of Lemma 7. By Lemma 2 every $F \in \mathscr{F}$ has a (uniformly) approaching anti-cover $\mathscr{C}_{F}=\left\{V_{\lambda}: \lambda \in \Lambda(F)\right\}$. Since $\operatorname{dim}(T-g(p(Y))) \leqq n$ and 
$T$ is hereditarily paracompact, we can assume that $\mathscr{V}_{F}$ is locally finite in $T-F$ and $\operatorname{dim} B_{T-F}\left(V_{\lambda}\right) \leqq n-1$ for every $\lambda \in \Lambda(F)$. Let $\Delta(F)$ be the totality of subsets $\delta$ of $\Lambda(F)$ such that

$$
V(\delta)=F \cup\left(\mathbf{U}\left\{V_{\lambda}: \lambda \in \delta\right\}\right)
$$

is an open neighborhood of $F$ in $T$. Then we have $\operatorname{dim} B(V(\delta)) \leqq$ $n-1$ for every $\delta \in \Delta(F)$. Set

$$
\begin{aligned}
& \mathscr{F}(F)=\{V(\delta): V(\delta) \subset H(F), \delta \in \Delta(F)\}, \\
& \mathscr{C}_{i}=\bigcup\left\{\mathscr{T}(F): F \in \mathscr{F}_{i}\right\}, \\
& \mathscr{U}_{1}=\bigcup\left\{\mathscr{V}_{i}: i \in N\right\}, \\
& \mathscr{W}=\mathscr{W}_{0} \cup \mathscr{W}_{1} .
\end{aligned}
$$

Then $\mathscr{W}$ is the required base for $T$.

TheOREM 3. If $Y$ is a closed image of a paracomplex, then $Y$ satisfies $(A)$.

Proof. Since by Theorem 1 is an $M_{1}$-space, the if part of $(A)$ is nothing but [5, Lemma 7]. Let $f: X \rightarrow Y$ be a closed mapping of a paracomplex $X$ onto $Y$ with $\operatorname{dim} Y \leqq n$. We use the same denotion as in the proof of Theorem 1. By Lemmas 8 and 9 , we can take each $U\left(\alpha_{1}, \cdots, \alpha_{i}\right)$ so that

$$
\operatorname{dim} B_{f\left(X_{i}\right)}\left(U\left(\alpha_{1}, \cdots, \alpha_{i}\right)\right) \leqq n-1 .
$$

From the construction of $U\left(\alpha_{1}, \cdots, \alpha_{i}\right)$, we have $\operatorname{dim} B\left(U_{\mu}\right) \leqq n-1$ for every $\mu$ because

$$
B\left(U_{\mu}\right) \subset \bigcup_{i=1}^{\infty} B_{f\left(X_{i}\right)}\left(U\left(\alpha_{1}, \cdots, \alpha_{i}\right)\right), \mu=\left(\alpha_{1}, \alpha_{2}, \cdots\right) .
$$

This completes the proof.

Acknowledgment. I would like to thank the refree for informing me that the main result, Theorem 1 , of this paper is independently proved by Gary Gruenhage in his new paper "On the $M_{3} \Rightarrow M_{1}$ question", the result of which states that the closed image of a stratifiable $\mathscr{F}$-metrizable space is $M_{1}$.

\section{REFERENCES}

1. C. J. R. Borges, On stratifiable spaces, Pacific J. Math., 17 (1966), 1-16.

2. J. G. Ceder, Some generalizations of metric spaces, Pacific J. Math., 11 (1961), 105-125.

3. T. Mizokami, On Nagata's problem for paracompact $\sigma$-metric spaces, Top. Its Appl., 11 (1980), 211-221. 
4. K. Nagami, The equality of dimensions, Fund. Math., 106 (1980), 239-246.

5. J. Nagata, On Hyman's M-spaces, Topology Conference (Virginia Polytech. Inst. and State Univ., 1973), 198-208.

6. F. G. Slaughter, The closed image of a metrizable spaces is $M_{1}$, Proc. Amer. Math. Soc., 37 (1973), 309-314.

Received October 30, 1980.

Sasebo Technical College

Sasebo, Nagasaki 857-11,

JAPAN 



\section{PACIFIC JOURNAL OF MATHEMATICS}

\section{EDITORS}

DONALD BABBITT (Managing Editor)

University of California

Los Angeles, California 90024

Hugo RossI

University of Utah

Salt Lake City, UT 84112

C. C. MOORE and ANDREW OGG

University of California

Berkeley, CA 94720

\section{J. DugundJI}

Department of Mathematics University of Southern California Los Angeles, California 90007

R. Finn and J. Milgram Stanford University

Stanford, California 94305

\section{ASSOCIATE EDITORS}
R. ARENS
E. F. BeCKENBACH
B. H. Neumann
F. WoLF
K. YoSHIDA

\section{SUPPORTING INSTITUTIONS}

UNIVERSITY OF ARIZONA

UNIVERSITY OF BRITISH COLUMBIA

CALIFORNIA INSTITUTE OF TECHNOLOGY

UNIVERSITY OF CALIFORNIA

MONTANA STATE UNIVERSITY

UNIVERSITY OF NEVADA, RENO

NEW MEXICO STATE UNIVERSITY

OREGON STATE UNIVERSITY
UNIVERSITY OF OREGON

UNIVERSITY OF SOUTHERN CALIFORNIA

STANFORD UNIVERSITY

UNIVERSITY OF HAWAII

UNIVERSITY OF TOKYO

UNIVERSITY OF UTAH

WASHINGTON STATE UNIVERSITY

UNIVERSITY OF WASHINGTON 


\section{Pacific Journal of Mathematics}

\section{Vol. 97, No. $1 \quad$ January, 1981}

Charles A. Asmuth and Joe Repka, Tensor products for $S L_{2}(\mathfrak{k})$. II.

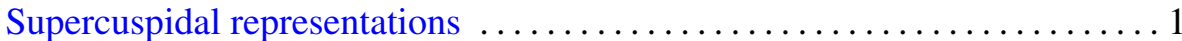

Joseph Barback, On finite sums of regressive isols . ................. 19

Matthew G. Brin and Daniel Russell McMillan, Jr., Generalized

three-manifolds with zero-dimensional nonmanifold set ............29

Kun Soo Chang, Converse measurability theorems for Yeh-Wiener space . . . 59

Christopher Brian Croke, A "maximal torus" type theorem for complete

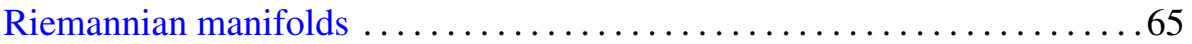

Gustave Adam Efroymson, Sums of squares in planar Nash rings . . . . . . 75

John Robert Fisher, Axiomatic radical and semisimple classes of rings . . . .81

Betty Kvarda, Consecutive integers for which $n^{2}+1$ is composite .......93

Roosevelt Gentry, New diagram proofs of the Hausdorff-Young theorem

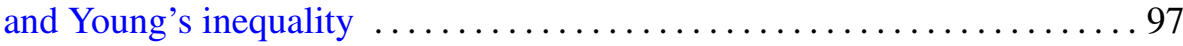

Patrick M. Gilmer, Topological proof of the $G$-signature theorem for $G$

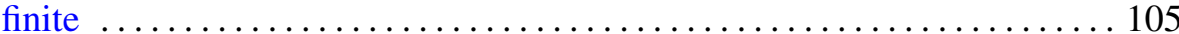

Chung Wei Ha, A noncompact minimax theorem .................. 115

James J. Hebda, Manifolds admitting taut hyperspheres ................ 119

Takayuki Kawada, Sample functions of Pólya processes ............. 125

Peter K. F. Kuhfittig, Common fixed points of nonexpansive mappings by

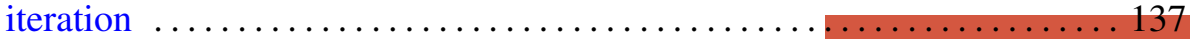

James Thomas Loats and Judith Roitman, Almost rigid Hopfian and dual

Hopfian atomic Boolean algebras .......................... 141

Roger McCann, On embedding semiflows into a radial flow on $l_{2} \ldots \ldots \ldots 151$

John McDonald, Closed orbits of convex sets of operators on the disk

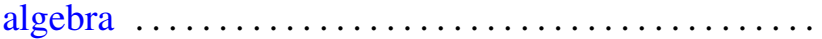

Mark D. Meyerson, Convexity and the table theorem .............. 167

Arnold William Miller, Generic Souslin sets . . . . . . . . . . . . . . . . 171

Takemi Mizokami, On the closed images of paracomplexes $\ldots \ldots \ldots \ldots \ldots 3$

Jagannadham Venkata Pakala and Thomas Stephen Shores, On

compactly packed rings $\ldots \ldots \ldots \ldots \ldots \ldots \ldots \ldots \ldots$

Andrew Pletch, Strong completeness in profinite groups

Wilbur Carrington Whitten, Inverting double knots

James Juei-Chin Yeh, Existence of strong solutions for stochastic

differential equations in the plane 